\title{
DOCENCIA E INNOVACIÓN EN LA UNIVERSIDAD: DE CONCEPCIONES Y PRÁCTICAS "VIVIENTES"
}

\section{TEACHING AND INNOVATION AT THE UNIVERSITY: OF "LIVING" CONCEPTIONS AND PRACTICES}

\author{
Erika Yamila Sánchez ${ }^{1}$ - Patricia Belén Demuth ${ }^{2}$ - Mara Elisabet Moreyra ${ }^{3}$
}

Fecha de recepción: 24-06-2019.

Fecha de aceptación y versión final: 21-11-2019.

\section{Resumen}

El siguiente artículo focaliza en los resultados de investigación sobre la docencia universitaria, particularmente en el análisis de un caso contextualizado en una asignatura de la carrera de Arquitectura reconocida como una práctica pedagógica innovadora de la Universidad Nacional del Nordeste. Nuestro principal interés radica en comprender los fundamentos de prácticas pedagógicas innovadoras construidos por los profesores que las desarrollan, a través de su ejercicio profesional. En términos metodológicos, se enmarca en un paradigma interpretativo de investigación y en un enfoque cualitativo, valiéndose del estudio de casos. Los instrumentos de recolección de datos fueron micro-entrevistas antes y después de las clases, entrevistas en profundidad, observaciones de clases y análisis de documentación curricular: programa y plan de estudios. Como conclusiones eminentes, señalamos la reconstrucción de un caso de docencia universitaria cuya propuesta pedagógica se sustentó en una comunidad de escasos recursos económicos, que actuó como escenario de la propuesta de aprendizaje desde una perspectiva crítica, relacionando un "laboratorio viviente", la dimensión disciplinar, el rol activo y de autogestión estudiantil y la evaluación de aprendizajes.

Palabras clave: práctica pedagógica - Innovación - estrategias de enseñanza - Arquitectura.

\section{Abstract}

The following article focuses on the results of research on university teaching, particularly in the analysis of a case contextualized in a subject of the Architecture career recognized as an

\footnotetext{
${ }^{1}$ Profesora y Licenciada en Ciencias de la Educación. Esp. en Educación y NTIC. Auxiliar Docente de Primera en Didáctica- Facultad de Ciencias Exactas y Naturales y Agrimensura, Universidad Nacional del Nordeste (UNNE). Auxiliar Docente de Primera en Didáctica II, Facultad de Humanidades (UNNE). Domicilio: $B^{\circ}$ Guiraldes Qta. $1 \mathrm{mz} 12$ Dpto. 01-10. Resistencia, Chaco, República Argentina. Tel. celular: 3794-702355. Correo electrónico: erikayamilasanchez@gmail.com

${ }^{2}$ Doctora en Didáctica y Organización de Instituciones Educativas (Universidad de Sevilla), España. en Cs. Sociales (FLACSO). Profesora y Licenciada en Ciencias de la Educación. Profesora Titular en Pedagogía Universitaria y Tecnología Educativa, Facultad de Medicina, Universidad Nacional del Nordeste (UNNE). Auxiliar Docente de Primera Didáctica I, Facultad de Humanidades (UNNE). Domicilio: Madariaga 2124. Corrientes, República Argentina. Tel. celular: 3794-388200. Correo electrónico: patriciademuth@ hotmail.com

3 Profesora y Licenciada en Ciencias de la Educación. Becaria Doctoral CONICET-Facultad de Humanidades, Universidad Nacional del Nordeste (UNNE). Domicilio: San Martin 1414, $3^{\circ}$ p. Dpto. 12. Resistencia, Chaco, República Argentina. Tel. celular: 3624-547945. Correo electrónico: maraunne@ hotmail.com
} 
innovative pedagogical practice of the Universidad Nacional del Nordeste. Our main objective is to understand the foundations of innovative pedagogical practices built by teachers through their professional practice. Methodologically, it works from an interpretative paradigm and a qualitative approach, with case studies. The data collection instruments were micro-interviews before and after the classes, in-depth interviews, observations of non-participating classes and analysis of curricular documents. As conclusions we point out the reconstruction of a case of university teaching whose pedagogical and didactic proposal was sustained in a community of scarce economic resources, which acted as a scenario of the didactic proposal from a critical perspective, relate a "living laboratory", the disciplinary dimension, the active student role and the evaluation of authentic learning.

Key words: pedagogic practice- Innovation- teaching strategies- Architecture.

\section{Introducción}

El presente artículo trabaja sobre el análisis y los resultados de investigación de un caso único (Yin, 2004; Stake, 2007), de docencia universitaria en el que se evidencia la influencia de las concepciones y conocimientos didácticos de un equipo docente en la propuesta de una asignatura de la carrera de Arquitectura identificada como innovadora en el contexto de la Universidad Nacional del Nordeste. La relevancia del presente escrito y el objetivo de nuestra indagación, radica en la posibilidad de comprender que toda práctica pedagógica innovadora se fundamenta en conocimientos y concepciones didácticas y epistemológicas que los profesores construyen a través de su ejercicio profesional.

En este sentido, nuestro interés por indagar sobre el conocimiento profesional docente de profesores universitarios de la Universidad Nacional del Nordeste, las concepciones que las fundamentan, la caracterización de las estrategias metodológicas y sus fundamentos; se sostiene en la posibilidad de identificar casos de docencia universitaria valiosos para ser analizados y sistematizados, enriqueciendo el acervo del campo de estudio de la Didáctica de Nivel Superior.

Esta línea de investigación centrada en el conocimiento didáctico del contenido de docentes universitarios y su relación con las prácticas pedagógicas que realizan en el contexto de las asignaturas para la formación profesional, se desarrolla en varios proyectos de investigación del Grupo CyFOD (Conocimiento y Formación Docente) de la UNNE desde el año 2008. Específicamente, esta indagación se encuadra en el paradigma interpretativo de investigación, el enfoque cualitativo, y bajo la estrategia metodológica "estudio de casos". Concretamente, este caso fue construido a partir de 3 entrevistas en profundidad al equipo docente, variadas micro-entrevistas antes y después de las clases, observaciones de clase (presenciales y en tareas en terreno) durante un período de dos meses, y análisis de los contenidos de documentos curriculares de la asignatura y la carrera.

En cuanto a los resultados, trabajamos desde la reconstrucción del caso a partir de su desarrollo narrativo y señalamos como conclusiones más destacadas la recuperación de un caso de docencia universitaria innovadora, centrada en el estudiante y en la comunidad social más desfavorecida, en la que se integra, desde una perspectiva crítica de la profesión, el conocimiento disciplinar, y el planteo pedagógico; el sentido de trabajar en un "laboratorio viviente" sensible con la realidad 
circundante, que atiende las necesidades concretas de la realidad, y transforma en los procesos de aprendizaje, enseñanza y evaluación a los estudiantes, a los mismos profesores que diseñan las prácticas pedagógicas y a las investigadoras, que lo transitamos.

\section{Marco teórico}

En nuestra intención por indagar las "buenas" prácticas pedagógicas (Porta 2011; 2016) que involucran al docente universitario, intentamos conocer en profundidad aquellas que se consideran como innovadoras dentro de la universidad, entendidas como prácticas que generan cambios, pensados para mejorar y brindar logros pedagógicos que modifiquen las prácticas tradicionales, favoreciendo el desarrollo de aprendizajes profundos en las aulas y para la formación profesional.

El fenómeno de la innovación universitaria, fue considerado en el pasado como un elemento individual, esporádico y aislado, pasa a ser ahora un fenómeno social y colectivo, en el que la universidad debe desempeñar un papel clave; tiene que conjugar sus actividades científicas y tecnológicas con otras de tipo organizacional, financiero y comercial, orientadas a transformar profundamente las estructuras generales y productivas de cualquier sociedad contemporánea desarrolladas o en vías de desarrollo (Casas \& Stojanovic, 2016). Investigaciones en este campo, manifiestan la necesidad de que la cultura y las estructuras organizativas de las instituciones educativas deben estar en línea con las nuevas formas de trabajo colaborativas para la generación de un entorno consciente que facilite el trabajo de los docentes innovadores para la integración de la innovación (Kunnari et al., 2016).

Por ello, creemos de gran importancia considerar a las prácticas pedagógicas innovadoras como actividades que no están aisladas, que dependen del acompañamiento de toda la institución y que necesitan ser estudiadas para comprenderlas y promoverlas para el logro de desarrollos pedagógicos sobresalientes.

Desde la perspectiva de Pérez Ortega (2016), la innovación educativa es un conjunto de ideas, procesos y estrategias, más o menos sistematizados, mediante los cuales se trata de introducir y provocar cambios en las prácticas educativas vigentes. La innovación no es una actividad puntual sino un proceso, un largo viaje o trayecto que se detiene a contemplar la vida en las aulas, la organización de los centros, la dinámica de la comunidad educativa y la cultura profesional del profesorado. Su propósito es alterar la realidad vigente, modificando concepciones y actitudes, alterando métodos e intervenciones y mejorando o transformando, según los casos, los procesos de enseñanza y aprendizaje. La innovación, por tanto, va asociada al cambio y tiene un componente -explícito u oculto ideológico, cognitivo, ético y afectivo; apela a la subjetividad del sujeto y al desarrollo de su individualidad, así como a las relaciones teoría-práctica inherentes al acto educativo (Carbonell, 2002).

Siguiendo esta línea se sostiene que innovar es aplicar tres condiciones importantes en todo ejercicio profesional: apertura, actualización y mejora. La apertura está unida a la flexibilidad y capacidad de adaptación, no se trata de cambiar algo rígido por otra cosa igual de rígida. La actualización tiene que ver con la puesta a punto, cuando se introducen nuevos modelos y/o formas de actuación. Se trata de poner al día los sistemas de enseñanza, incorporando los nuevos conocimientos y 
recursos disponibles y la mejora es el compromiso de toda innovación para no caer en retrocesos (Zabalza, 2004).

Creemos en la importancia de un docente innovador en el proceso de innovación educativa; consideramos, que los profesores son los actores decisivos de los procesos de cambio educativo y de las innovaciones, tanto pedagógicas como tecnológicas y sólo son viables si cuentan con la complicidad y el protagonismo de los docentes (Michavila, 2009).

Es así que, conocer más acerca del docente universitario, del docente innovador, "capaz de dedicar mucho tiempo en olvidarse de las modas pedagógicas y dejarse guiar por su sentido común pedagógico sobre cómo lograr que el aprendizaje genuino efectivamente se logre y hacer de la innovación una práctica cotidiana (...), capaz de mostrar a otros cómo enseña, que muestra con orgullo su creación, que estudia y que necesita nutrirse de la historia de la educación reciente y remota para saber lo que otros ya han pensado y han hecho en las aulas" (Libedinsky, 2001), es nuestra principal motivación y objetivo.

En esta oportunidad, centraremos la mirada en un equipo docente del campo de la arquitectura, considerado innovador y cualificado por estudiantes y autoridades institucionales como práctica valiosa en el campo de la formación de arquitectos.

Concretamente, en diferentes artículos que abordan la formación en arquitectura, se reconoce la necesidad de atender la demanda de un nuevo perfil para arquitectos, integrando y fomentando las correspondientes competencias a través de nuevas metodologías de aprendizaje, herramientas y conceptos, principalmente orientadas al desarrollo sostenible de las construcciones, con fuerte compromiso y responsabilidad social por parte de la universidad hacia la sociedad para el desarrollo de soluciones innovadoras de los conflictos ambientales, sociales y económicos. En el campo específico se reconoce la urgencia de plantear nuevos procesos en la enseñanza (Masseck, 2017) puesto que, continuamente surgen nuevas demandas en dicho campo de formación vinculadas a la realidad local, internacional, métodos de cálculos, requerimientos para la sustentabilidad ambiental, que esencialmente reclama un perfil profesional direccionado por competencias que atraviesen el curriculum (Jiménez, 2010).

Un ejemplo concreto es la Universidad Laica "Vicente Rocafuerte", de la República del Ecuador, quien introdujo como parte de la propuesta curricular de la carrera de arquitectura la integración del método de aprendizaje basado en proyectos, reconociendo la emergencia de los nuevos escenarios sociales y educativos atravesados por las Nuevas Tecnologías de la Información y Comunicación (TIC) y las exigencias en la calidad del perfil profesional que demandan el desarrollo de competencias particulares para la calidad de una formación competente (Pino Medrano, 2015).

Además de iniciativas internacionales que en estos últimos años se han realizado sobre la formación en la educación superior, particularmente en el área de arquitectura, diseño y urbanismo, referimos al Proyecto ALFA ADU_2020 desarrollado desde 2012-2014 y que fue parte del programa ALFA III. Un proyecto de cooperación entre países de América Latina y la Unión Europea que trabaja sobre el debate de la educación superior como canal para el desarrollo socio- económico y la lucha contra la inequidad socio-cultural. Dicho proyecto se realizó a través de un "Taller Internacional" 
realizado en la Universidad Católica de Santiago de Guayaquil (México) en el 2013, durante el periodo de veinte días consecutivos que involucró a profesores y estudiantes del 4to y 5to año de la carrera de arquitectura en universidades de Argentina, Bélgica, Bolivia, Brasil, Panamá, Cuba, UK, entre otros (Ricol y Zurita, 2014). El trabajo consistió en la socialización y confrontación de experiencias devenidas de las propuestas didácticas de los docentes y el trabajo de los estudiantes, comparando metodologías de enseñanza tradicionales, abrir el debate sobre la incorporación de nuevas estrategias de enseñanza de manera sistemática a nivel curricular, que vincule a las comunidades sociales, las necesidades locales y las competencias profesionales esenciales que exige el contexto socio cultural del siglo XXI. Asimismo, repensar el sistema de evaluación en las universidades involucradas en el proyecto, advirtiendo así una iniciativa que genera expectativas sobre nuevas formas de hacer y ser en el perfil del arquitecto.

El trabajo innovador y la tendencia de formación en el campo de la arquitectura, refiere a la construcción de "Living Labs", que pueden verse como ecosistemas sociales o Arenas de Innovación con un vínculo al contexto socioeconómico circundante, fomentando la creación de comunidades educativas transversales (Masseck, 2017), como se podrá observar en el caso de nuestros protagonistas.

Otras de las revisiones bibliográficas en esta dirección, manifiesta que a través de estos "laboratorios vivos" los estudiantes desarrollaron una variedad de habilidades y técnicas que fueron valiosas para sus proyectos de graduación y que luego los apoyarán como arquitectos en el futuro. Pudieron experimentar las condiciones climáticas y la tecnología de construcción en un contexto específico y asociados con la arquitectura vernácula local específico. Dicha experiencia se utilizó para desarrollar nuevos enfoques de arquitectura climática adaptativa y receptiva por medio de pruebas físicas en modelos de células de prueba en un entorno de laboratorio vivo (Dabaieh et al., 2017).

\section{Marco metodológico}

Este trabajo se inscribe en la investigación denominada "La construcción del conocimiento didáctico del contenido en profesores experimentados y principiantes de la UNNE. Estudio de casos", acreditada por la Secretaría General de Ciencia y Técnica de la UNNE, con inicio en el año 2015 y finalización en el año 2019.

Se encuadra en el paradigma interpretativo y el enfoque cualitativo, se buscó indagar para la comprensión de las concepciones docentes, las características de las prácticas pedagógicas innovadoras de un equipo docente de una asignatura de la carrera de Arquitectura: Gestión y Desarrollo de la Vivienda Popular, ubicada en el quinto año del Plan de Estudios de la carrera.

Este tipo de estudio no pretende generalizar de manera intrínseca resultados de poblaciones más amplias, ni necesariamente obtener muestras representativas (bajo la ley de probabilidad); inclusive, no busca que llegue a refutarse. Asimismo, se trata de fundamentar más un proceso inductivo y se pretende ir de lo particular a lo general (Hernández Sampieri, Fernández Collado y Baptista Lucio, 2003).

De este modo se buscó no solo conocer las concepciones de las prácticas pedagógicas innovadoras de docentes universitarios de la UNNE, si no también 
describir y fundamentar las características que poseen y qué utilidad se le atribuyen, el cambio en los aprendizajes de los estudiantes evidenciados por los docentes, como así también el vínculo con la investigación educativa.

\subsection{El proceso de selección}

Siguiendo las condiciones y el contexto en el que se desarrolló la naturaleza de nuestra indagación se trató de una investigación de estudio de caso de 1 docente experimentado con su equipo docente en la Facultad de Arquitectura de la UNNE, el mismo fue seleccionado a través de criterios vinculados con la trayectoria profesional, las referencias institucionales y las prácticas de investigación en la disciplina. Las fuentes de información fueron documentales y testimoniales. Porque es la estrategia metodológica más adecuada a las particularidades del objeto, ya que ofrece un estudio descriptivo profundo de la situación que se pretende estudiar (Gewerc y Montero, 2000).

\subsection{Criterios de selección del caso}

Profesor experimentado que presentó trabajo en las Jornadas de Comunicación de Experiencias Pedagógicas de la UNNE, entendiendo a los mismos (trabajos) como evidencias concretas de la tradición en innovación que representan, dichos trabajos han sido sometidos a referato externo, y por una serie de revisiones de comités académicos, en este sentido, son representativos de las mejores prácticas de innovación de la UNNE.

El estudio de casos es particularmente apropiado para el estudio de situaciones que requieren un notable grado de intensidad y, normalmente, un reducido período de intervención. El estudio de casos nos permitiría el análisis profundo de lo que sucede en los casos hacia el interior de los mismos y hacia sus contextos buscando en la síntesis la construcción de significado (Demuth Mercado, 2011).

La asignatura y el desarrollo de clases estaban conformadas por una docente titular a cargo que cumplía con estos requisitos enunciados; su equipo lo conformaban: un profesor adjunto, tres auxiliares docentes y cinco profesionales adscriptos del campo de la Arquitectura, y la presencia de una docente invitada de campo del Derecho. La matrícula de cursado superaba los 180 cursantes.

Figura 1: "Etapas del diseño de la investigación"
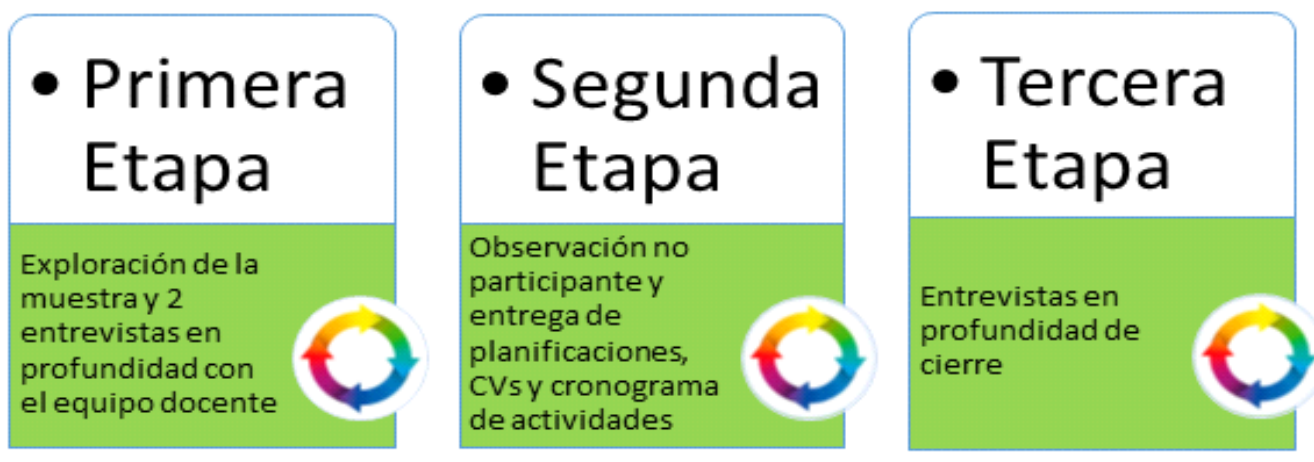

Fuente: Elaboración propia.

Revista del Instituto de Investigaciones en Educación

ISSN $1853-1393$

Resistencia - Chaco - Argentina 
Existieron cuatro fuentes de recolección de la información para la metodología de casos: los documentos, las entrevistas, observación y observación participante, donde el investigador recurrirá a un tipo de fuente o a otro dependiendo de la naturaleza del estudio, de las informaciones que se quieran obtener y de la accesibilidad de la información (Coller, 2005).

En nuestro estudio se analizaron la información con tres fuentes mencionadas: los documentos institucionales, materiales brindados informantes clave de las facultad, de los profesores seleccionados se analizaron sus materiales personales y didácticos, datos sobre el rendimiento académico de los alumnos acerca del impacto de las prácticas, los materiales de evaluación que utilizaron estos profesores, las experiencias de los aprendizaje de los estudiantes y los aspectos identificados en las entrevistas precedentes y las observaciones de clases.

Las entrevistas en profundidad como estrategia de recogida de información, que consistió en recoger datos sobre acontecimientos y aspectos subjetivos del profesor que otras técnicas existentes no permitieron, además nos permitió acercarnos al escenario que queríamos estudiar de manera más accesible, menos establecida. Las observaciones de clases no participante se realizaron de un ciclo temático (inicio, desarrollo y cierre).

\section{Análisis y Discusión}

En términos generales, las prácticas se caracterizaron por poseer momentos: un primer momento, consistieron en la presentación del tema con el que iban a trabajar en el día, un segundo momento: de trabajo grupal y un tercer momento, donde realizaban una puesta en común.

El equipo docente estaba constituido por seis profesores arquitectos, una abogada y una asistente social, este año la asistente social no estaba presente en su totalidad, pero tenían un vínculo muy cercano y una estudiante adscripta.

El equipo fuera del aula se desempeñaba laboralmente en ámbitos tradicionales de desempeño de la arquitectura, desde propias empresas o en el ámbito estatal, sólo la docente titular tenía una dedicación más profunda con la docente. A continuación, se desarrollarán los diferentes análisis de las categorías teóricas implicadas:

\subsection{Estrategias de enseñanza}

Las estrategias de enseñanzas definidas como actividades realizadas por los docentes y los estudiantes que permitieron un proceso de análisis de una conferencia, de casos, de una película y la simulación de una entrevista como herramienta de investigación para el desarrollo de procedimientos prácticos, a través de estos escenarios, los docentes introducían a los estudiantes en un campo del desarrollo profesional por medio de un trabajo realizado en el aula y en terreno, ubicados en reconocidos asentamientos de la ciudad de Resistencia.

En este sentido, consideramos a las estrategias metodológicas como actividades que permitieron apropiarse de los conocimientos a través de procesos constructivos por parte de los alumnos. En ellas se integran tanto cuestiones 
disciplinares (aspectos teóricos y metodológicos) del saber a enseñar, como de aprendizaje (procesos reflexivos, de conflicto y superación de contradicciones, de análisis y síntesis, de juicio y argumentación, etc.), que cada docente debe haber vivenciado él mismo para plantear luego a sus alumnos una propuesta similar de reelaboración del conocimiento (Litwin, 2002).

La primera estrategia desarrollada consistió en una introducción por medio de vídeos de autores referentes de la arquitectura que explicitaban temas relevantes que iban a profundizar en el cursado, donde después de observarlos se generó un intercambio de reflexiones acerca de lo que había observado. De acuerdo con estos aportes, el aprendizaje significativo sólo es posible cuando los alumnos relacionan, en forma consciente y activa, las nuevas informaciones con sus conocimientos y experiencias previas, y comprenden (reconstruyen) el significado del nuevo conocimiento, es por ello que la conferencia se presentó como organizadores previos, que implicó la presentación de un breve material introductorio del contenido de enseñanza y reunió los núcleos centrales de la asignatura (Davini, 2008).

Luego de mirar las conferencias se generaba un intercambio donde salían a la luz ciertos aspectos que traían los cursantes, referidos a poder ir a terreno a vivenciar las problemáticas vinculadas al desarrollo habitacional y así, poder identificar posibles soluciones.

Como segunda estrategia se logró identificar al análisis de casos. El equipo docente les brindó 3 casos que fueron noticia nacional acerca de problemas habitacionales y la bibliografía que la debían traer leídas para el día de la fecha, en donde la docente dividió el grupo clase en 3 , invitándolos a que cada grupo debía analizar un caso. Debían leer los casos, analizarlos con los textos que ya debían haberlos leídos, y luego debían presentar las producciones a sus compañeros a modo de exposición.

Este tipo de estrategia correspondió una forma natural de aprender alrededor de situaciones realistas que la vida diaria nos presenta, muestra e incluye distintas situaciones sobre las cuales reflexionamos, discutimos y tomamos alguna posición (al leer el periódico, escuchar la radio o televisión, hablar con los vecinos o intercambiar con los miembros del equipo de trabajo). Muchas veces, estas situaciones inclusive nos impulsan a buscar más información, expresar nuestras ideas o puntos de vista, o influir en el curso de la situación. Asimismo, pueden llevarnos a reflexionar sobre enfoques sistemáticos o conocimientos científicos, para comprender mejor la situación.

El método de estudio de casos recupera este proceso natural de entender, interpretar e intervenir en la realidad y lo sistematiza para la enseñanza. De esta forma, apuntó a vincular el conocimiento, la realidad y las prácticas, a través de la presentación de una situación de la vida real o prefigurada (lo más parecida posible a una situación real) como punto de partida para el aprendizaje.

En las estrategias mencionadas, las intervenciones de la docente jugaron un papel fundamental, asentándose en la curiosidad, el asombro, la búsqueda de explicación y comprensión.

De acuerdo con Perkins (1993), el análisis de casos constituye un "kit de construcción": incluyen una descripción del caso o situación y del contexto en el que

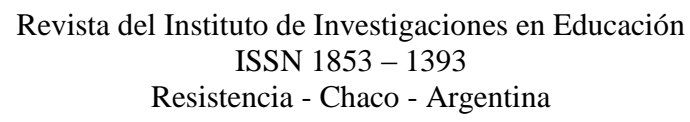


ocurre, el intercambio con el grupo generando preguntas y debate. Según sea el caso, se puede buscar informaciones sobre aspectos críticos de la situación (incluyendo posibles consultas bibliográficas" o a bases de datos), agregar entrevistas a otros actores o especialistas, recoger información sobre otros casos semejantes, clasificar las distintas visiones sobre el problema y diferentes testimonios, etc., dependiendo del grado de amplitud o profundidad de la situación. Que es lo que se logró realizar en clases siguientes.

Davini (2008) nos ayudó a comprender cómo el análisis de los casos y el intercambio que se produjo luego facilitó el desarrollo de habilidades cognitivas específicas, como la interpretación reflexiva, el intercambio intelectual con otros. Generándose así también un diálogo reflexivo con los pares y el docente mediante las intervenciones que les realizaba.

Otra estrategia de enseñanza que se logró identificar fue la simulación de una entrevista, donde se les mencionó a los estudiantes que la intención de la actividad es preparar la herramienta de recolección de información en el trabajo de campo. La simulación escénica, que consistió en la organización de un juego de roles o una dramatización de una situación determinada. Para ello, se organizó el escenario y las escenas en forma similar a una situación real, en la cual los alumnos desempeñaron distintos roles.

Otra de las estrategias de enseñanza que se logró identificar fue un cine debate, la docente mencionó que el objetivo de la actividad es para incorporar insumo para las preguntas que realizaron para realizar la entrevista. La película se caracterizaba por mostrar cómo viven las personas con problemas habitacionales, hacinamiento y pobreza. Luego de finalizar la docente les solicitó que trabajen en grupo y luego hicieron una puesta en común, donde participaron la gran mayoría de los estudiantes.

Luego del trabajo de campo que se realizó en los asentamientos de la Ciudad de Resistencia: Barrio Niño Jesús, Vista Linda, Fortaleza y 12 de agosto. Los estudiantes volvieron a la facultad, donde se pudo identificar una puesta en común acerca de lo que les ocurrió en su visita. La docente arrojaba disparadores para que participen todos: "Hoy fuimos a conocer una realidad, que no está alejado de nuestra cotidianeidad, por eso me gustaría que ustedes mencionen con qué se encontraron, si fueron y con la idea de encontrarse con una casa, con ciertas personas, etc."

De este modo, los estudiantes respondían que las características que más sobresalieron fue la precariedad con la que viven, que poseen diversas necesidades, muchos jóvenes que se drogan y lo comparaban con barrios que están cerca de sus casas, ejemplo con el barrio La olla de Corrientes.

\subsection{Estrategias de aprendizaje}

Luego de la descripción de las estrategias de enseñanza, se puede identificar que las estrategias de aprendizaje que se observaron, en el análisis de la conferencia, del caso de la película fueron el trabajo en grupal, de análisis, debate y puesta en común de las conclusiones que abordaron. Anijovich (2009) nos plantea que teniendo en cuenta el escenario que fue diseñado decidiendo las estrategias de enseñanza que fueron analizadas en el apartado anterior, las estrategias de aprendizaje que se

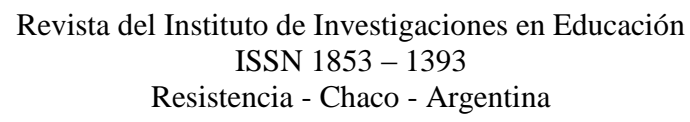


realizaron en la experiencia, promovieron en los estudiantes procesos interactivos, entre lo nuevo que se les presentó y lo que ellos traían.

Consideramos que los estudiantes se apropiaron tanto del conocimiento disciplinar como de las habilidades cognitivas asociadas a ellos y pudieron transferir todo lo construido a su trabajo en terreno, que fue lo más cercano al desarrollo de la práctica profesional.

Los estudiantes trabajaban en grupos de aproximadamente 10 alumnos por grupo, donde se dividían las actividades de manera deliberada, distribuyendo el tiempo que les designaban la docente, donde utilizaban el tiempo para expresar lo que pensaban acerca del tema a trabajar, que aportes teóricos brindarían acerca de las cuestiones observadas llevando así a la puesta en común con el grupo clase. Esto invitaba a que los otros grupos participen y debatan incorporando cuestiones que lo otros grupos pasaron por alto: nos hace pensar el arquitecto, sin embargo, es el cambio de la cultura y de ahí se empieza a cambiar ciertos parámetros que influyen, porque él decía que no solucionamos todo brindándole una casa, sino que ahí comenzaba todo, pero todos tenemos que brindar para contribuir a esa ciudad democrática y eso se lograría con la participación de todos los actores sociales.

El aprendizaje se construyó, mediante que los alumnos y las alumnas elaboraron, mediante la actividad personal por medio de interacción con el grupo de pares y las intervenciones de la docente, los conocimientos culturales. Por todo ello, la enseñanza consiste en prestarle al alumnado la ayuda que necesita para que vaya construyéndolos (Mauri, 1999). En la simulación de una entrevista, un grupo de alumnos representaría a las familias, otro a los entrevistadores y el resto del grupo clases representaría a los observadores, dándoles indicaciones de que tienen que tener en cuenta para preguntar a las familias y que deben observar. La simulación consistió en varias entrevistas que fueron dramatizadas por varios grupos de alumnos, en las cuales interactuaban por medio de preguntas exploratorias, para conocer más acerca de la familia y de las condiciones habitacionales e históricas.

La vinculación con la realidad simulada, no fue automática, sino el resultado de un proceso activo del alumno y la alumna que le permitió, reorganizar el propio conocimiento y enriquecerlo. El alumno y la alumna necesitaron poseer toda una serie de destrezas metacognitivas que les permitió asegurar el control personal sobre sus conocimientos y los propios procesos durante el aprendizaje. El profesor se volvió un participante activo en el proceso de construcción de conocimiento que tuvo como centro no a la materia, sino al alumno y a la alumna que actúan sobre el contenido que han de aprender (Mauri, 1999).

Luego de la visita programada a los asentamientos de la Ciudad de Resistencia, lo alumnos volvieron a la Facultad y realizaron una puesta en común acerca de las cuestiones que les resultó interesantes. Esta puesta en común estuvo moderada por una docente invitada, abogada, que los invitaba a pensar a los alumnos acerca de cómo se sintieron al vincularse con la visita. La integración (Eggen et al., 1999) es el proceso de unir la nueva información a los conocimientos previos; las preguntas exploratorias que realizó la docente, alentaron a la integración horizontal, al pedir, por ejemplo, como se sintieron, que diferencia o semejanza tienen con lo que ellos ya conocían. 
Las realidades con las que se encontraron les ocasionó un gran impacto que les ayudó a reflexionar acerca de cómo redefinir esa problemática que ellos creían conocer, haberlas leído en un caso, escuchado en una conferencia, observado en una película, simulado en una entrevista. Reconocieron que el recorrido hasta llegar a la realidad los ayudó, pero que el impacto con la gente fue superador. Este cierre promovió la integración, al resumir y revisar todos los temas que fueron trabajados desde el inicio del cursado, remarcando los puntos importantes y proporciona las conexiones con los nuevos aprendizajes (Eggen et al., 1999).

Como se puede apreciar en ambas categorías, la diversidad de estrategias de enseñanza ha llevado a una multiplicidad de actividades y procesos de aprendizaje en los estudiantes, desde niveles conceptuales a niveles procedimentales complejos vinculados al campo de la arquitectura. En este sentido, Engeström retoma estos elementos que nos permiten comprender acerca de las actividades, siendo éstas “...las que se realizan mediante una constante negociación, orquestación y lucha entre las distintas metas y perspectivas de los estudiantes. El objeto y el motivo de una actividad colectiva son como un mosaico en constante evolución, una pauta que nunca se establece por completo" (Engeström, 1999 citado en Daniels, p.131).

Este planteo metodológico se puede caracterizar claramente como laboratorio viviente o "Living lab", ya que se constituye en un modelo de innovación social de experimentación y co-creación, con participación de usuarios y/o ciudadanos reales, en entornos reales (Bergvall - Kåreborna et al. 2009) en este caso, en el marco de una asignatura universitaria.

\subsection{Sistema de evaluación}

El sistema de evaluación se caracterizó por estar presente en todas las instancias de la clase, por medio de intervenciones que realizaba el docente a los estudiantes cuando realizaban sus trabajos en pequeños grupos y cuando les realizaba intervenciones cuando exponían en sus conclusiones al grupo grande de la clase, que fueron planificadas por el equipo docente. Es por ello que podremos decir, que el tipo de evaluación que se presentó en la experiencia, estuvo centrado en el logro de los objetivos educativos en detrimento de su metamorfosis cognitiva y metacognitiva (Tardiff, 2004).

Las intervenciones se hicieron presentes a modo de preguntas, abiertas, cerradas y exploratorias a los estudiantes que trabajaban bajo las consignas brindadas. En el caso de la evaluación que se les realizó cuando se analizó la conferencia, fue una evaluación diagnóstica acerca de los conocimientos previos que poseía el alumno. Ésta consistió en preguntas que se les realizó a modo de cuestionario y de intervenciones acerca algunos conceptos que ellos deberían traer de otras materias, como el concepto de complejidad que exponían de forma oral.

La evaluación diagnóstica es comúnmente asociada a un momento evaluativo inicial, en los primeros contactos con el grupo de alumnos, permitió valorar: las características socio-culturales de los estudiantes, sus capacidades, intereses y potencialidades, sus conocimientos previos y sus posibles dificultades. Esta valoración refiere tanto al grupo como conjunto y a los individuos particulares. 
En este sentido, la evaluación facilitó las decisiones previas del docente, a la hora de programar la enseñanza. En ello se incluye, también, la identificación de los recursos de enseñanza que pudieron ser movilizados y el análisis del contexto, entre otras importantes cuestiones. Se dirigió a detectar dónde estaban las dificultades de los alumnos y el porqué de las mismas. Buena parte de estas valoraciones se realizaron de modo informal y son continuas: interpretando las respuestas de los alumnos, sus percepciones y dificultades, analizándolas en función de los desafíos y el contexto personal inmediato. Dada esta informalidad, los profesores debieron estar atentos a ciertos riesgos (Davini, 2009).

El caso de la evaluación que se realizó cuando se analizaron los casos, por medio de intervenciones evaluaba la profundidad con la que analizaron los casos que les fueron asignados, consistió en realizarles preguntas acerca de cuestiones teóricas que ayudarían a interpretar los casos, el porqué de las decisiones que les llevó a las personas a llegar a estar en esas condiciones. Los alumnos se encontraban muy satisfechos con las devoluciones y aportes que les realizaba la docente.

Gardner (1992) define la evaluación como el proceso por el cual se obtiene información sobre los conocimientos (skills) y las capacidades (potentials) de una persona y, añade en la misma frase, que este proceso de recolección de información tiene como objetivo proporcionar una retroalimentación significativa a la persona a evaluar y proporcionar datos útiles a la comunidad en la que vive.

Cuando los estudiantes miraron la película los docentes evaluaban como analizaban los hechos que sucedieron en el grupo pequeño, ellos recorrían los grupos y realizaban intervenciones acerca de lo que tenían en cuenta. Eso llevo que al momento que compartieron sus conclusiones al grupo grande el docente se caracterizó como un moderador y los alumnos se posicionaban como protagonistas del debate acerca de las situaciones problemáticas que se lograron identificar.

Al momento de realizar la simulación de la entrevista los docentes evaluaban si se lograron realizar las intervenciones correspondientes en el entrenamiento como por ejemplo si se logró posicionar en el papel de los familiares, entrevistadores y observadores. Pero lo más interesante que se presentó, fue al momento de solicitar la docente, al grupo de observadores que les brinde una devolución a los otros estudiantes. De acuerdo con Meyer (1992), cualquier evaluación auténtica es necesariamente una evaluación de la competencia. Los problemas reales a resolver, ensayos, proyectos de investigación, simulaciones, actividades de laboratorio, etc. son situaciones de evaluación auténtica.

\subsection{Concepciones docentes}

Las concepciones docentes que se hicieron presente en las clases de arquitectura, reflejaron a la innovación como una actividad global de generación de cambios que construyeron a los aprendizajes reflexivos de los agentes del aula. Pero para contribuir a este tipo de aprendizaje, para que hubiera innovación necesito de innovadores; necesita de personas que se ilusionen, que se identifiquen y se comprometan con un proyecto que introduzca un cambio en sus prácticas habituales (Marcelo, 2013). 
La docente principal caracterizaba la importancia de incorporar este tipo de práctica, porque se encontraban con 180 alumnos que no tenían el hábito de la reflexión. Por eso creemos que el desafío de incorporar las estrategias anteriormente mencionadas, fue para fomentar un cambio en las prácticas que habitualmente desarrollaban los estudiantes. Webster \& Hopkins (1996). Esta mirada compleja de innovación, constituye una estrategia dirigida a aumentar los resultados de aprendizaje de los alumnos.

Según Viñao (2002, citado en Altopiedi, 2010), estas concepciones se vinculan a la innovación desde una mirada amplia, con las necesidades o problemas detectados en la enseñanza. Así, el sentido del término se restringe. No sólo se trata de una respuesta nueva, sino dirigida a solucionar aquellas dificultades registradas en la práctica. Se trata, por lo tanto, de la incorporación de modificaciones que afectan de forma puntual y directa a los procesos de enseñanza y de aprendizaje, en el ámbito de aprendizaje, para que los estudiantes se vinculen con el desarrollo de su práctica profesional.

Las concepciones docentes reflejaban la intención de incorporar prácticas innovadoras para generar un cambio en los estudiantes, por eso la actividad de incorporar el trabajo de campo, se la planifica muchos meses antes de iniciar el cursado, que consistió en concurrir a un asentamiento y conocer la realidad, recabar información que les sirvió de insumo para la realización de su trabajo final que consistió en dar una posible solución a la problemática. Pero incorporar estas prácticas no resultó nada fácil, se tuvo que realizar trabajos previos, con los referentes barriales, trabajar con las familias, explicándoles en que estas van a cumplir una función docente y que van a enseñar a un grupo de estudiantes de la universidad lo que es su realidad va a enseñar una realidad a los estudiantes.

La implementación con éxito de innovaciones en el aula es más probable que se produzca cuando los profesores suelen reflexionar acerca de su enseñanza y de los objetivos que persiguen. Por lo tanto, cuando las creencias pedagógicas son consistentes con las tecnologías los profesores se esfuerzan por utilizarlas para conseguir resultados positivos (Martín, 2009).

Los estudiantes ingresaron a la asignatura, con una forma de relacionarse con los docentes, sin embargo, al ingresar a vivienda se encontraron con un equipo de docente que realizaba un acompañamiento, evaluando cada avance que realizaba. La asignatura realizaba parciales, pero lo que les importaba, era que vayan construyendo poco a poco, día a día, que conozcan la pobreza cualitativamente.

Estas concepciones son consideradas como innovadoras, desde una mirada amplia, al sumergir en estas prácticas a los estudiantes a la realidad, de luego que los estudiantes de arquitectura al ingresar a la carrera, piensa que se va a dedicar su vida a la realización de hoteles, edificios, y estas prácticas ayudan a conocer su ciudad, las problemáticas que necesitan una solución. Libedinsky (2001) señala que:

....innovación educativa es sorpresa, consiste en solucionar problemas claramente detectados y a los que hay que dar respuesta, pero que a la vez nos posiciona en la tarea docente (...) como (...) transgresores. De aquellos que están convencidos de que el orden habitual y rutinario de la enseñanza debe ser alterado por nuevas formas de comunicación didáctica, por nuevas formas de entender y ejercer la enseñanza. (p.21) 
Para los docentes, estas prácticas se consideran como innovadoras porque para el alumno es una forma que no es común de aprender, están acostumbrados a trabajar con estructuras. Siendo el horizonte el trabajo interdisciplinario, porque consideran que es la única forma de enseñar a trabajar a los estudiantes y de abordar a la complejidad.

\section{Resultados}

Caso narrado: "El laboratorio viviente para la gestión y el desarrollo de la vivienda popular”.

Luego de haber recorrido y atravesado una experiencia significativa para nuestra formación en la investigación, nos aproximamos a la comprensión de un caso en el cual los actores involucrados promovieron a la innovación educativa como un cambio justificado, solucionando una alta gama de inconvenientes que se les presentaron en su práctica incorporando las estrategias metodológicas innovadoras para la disciplina de arquitectura y sus prácticas profesionales.

En el caso de Arquitectura, innovar fue introducir estos cambios justificados pero la calidad del cambio no dependió de lo valioso que fue el cambio en sí mismo sino de la justificación que se le atribuyó, una criteriosa justificación. Al considerar de suma importancia, mejorar la calidad de los aprendizajes de los estudiantes, para fortalecer sus prácticas profesionalizantes demandadas por esta sociedad.

Para introducir cambios, se necesitó de un tipo de profesores innovadores, porque como lo menciona Marcelo (2013) la innovación necesita de innovadores. Necesita de personas que se ilusionen, que se identifiquen y se comprometan con un proyecto que introduzca un cambio en sus prácticas habituales. En esta presentación, cumpliendo un rol importante del docente en relación a la calidad de los aprendizajes de los estudiantes. Los hallazgos más sobresalientes, que observamos en estas prácticas pedagógicas, fueron:

\section{- Docentes y estudiantes como protagonistas del proceso de enseñanza y aprendizaje}

Se articulaban las competencias del perfil profesional con los intereses de los estudiantes: se mezclaba lo que el profesor y la unidad académica interpretaban como necesario y lo que el alumno sentía como atractivo en ese momento, por ejemplo, vincularlo con la práctica profesional con casos frecuentes, con los que se van a enfrentar en la vida real.

\section{- Las planificaciones constituyeron una hipótesis de trabajo que se irá reformulando durante el proceso.}

El sistema de evaluación de estas experiencias, superaban una visión sumativa de medición de objetivos cumplimentado; concretizándose en un carácter de análisis procesual de las experiencias. Realizando un seguimiento de toda la experiencia.

Teniendo en cuenta la caracterización que poseen y reconocer que estos docentes se posicionan en una perspectiva crítico-reflexiva o dentro de un Enfoque 
Problematizador de la Enseñanza, como lo menciona Alcalá (2002), como un atenuante de las necesidades y problemas de la enseñanza.

Entienden que incorporar esta práctica innovadora, sirve para atenuar las necesidades y problemas detectados en la enseñanza, problemas que hacen referencia la exigua calidad de los aprendizajes de los estudiantes. Se cree que da una respuesta nueva, dirigida a solucionar aquellas dificultades registradas en la práctica. Modificando de forma puntual y directa a los procesos de enseñanza y de aprendizaje, en el ámbito del aula, para que los estudiantes se vinculen con el desarrollo de su práctica profesional.

Consideran que es la mejor forma para que los estudiantes aprendan, porque se habitúan en su formación a trabajar de manera muy estructurada, como su disciplina lo demanda, pero con estas prácticas consideran que es la mejor forma de ubicarlos en la realidad. Completamente involucrados, con el desarrollo de la investigación disciplinar, acerca del Desarrollo de la Vivienda Popular, que, al nivel Latinoamericano, tiene una gran repercusión esta línea de investigación disciplinar, por los índices de pobreza, por la ineficiente administración de los recursos de los Estados, y por la desvalorización de la Gestión de la Vivienda.

El valor de "sumergirse" con los estudiantes en la realidad para que conozcan la ciudad, las problemáticas, por ser una forma de aprendizaje que no es común para los estudiantes, porque están acostumbrados a agarrar un libro y trabajar con estructuras. Es así, que encontrarse con una realidad y diseñar una solución para mejorar la calidad de vida de los ciudadanos tan golpeados por el flagelo de la pobreza, estimulando su creatividad y despertando esa sensibilidad característica de esta disciplina, es tan necesaria para abordar temas tan delicados.

El argumento que empleaban para incorporar estas prácticas pedagógicas, fue pensar en los estudiantes, en la calidad de los aprendizajes y en despertar la reflexión de los estudiantes para que logren construir aprendizajes. En sus discursos los docentes, reflejan lo que Fenstermacher (1989), caracteriza como al docente libertador de la mente del individuo y un promotor de seres humanos morales, racionales, entendidos e íntegros. Matizado con argumentos característicos de las teorías constructivistas, las cuales integran diversas teorías que se han elaborado en oposición a la concepción asociacionista y mecanicista de psicología conductista. En esta posición nos hallamos ante un sujeto activo, no determinado exclusivamente por las contingencias ambientales, sino que interactúa con el medio desde sus experiencias y conocimientos anteriores, significando y resignificando el mundo que lo rodea, es decir realizando una verdadera reconstrucción de la realidad en sus estructuras mentales (Alcalá, 2002). Que consideran, como las más apropiadas para posicionarse al momento de diseñar sus propuestas; pensamiento construido, luego de atravesar una fuerte e enriquecedora formación docente, que la lograron y la siguen construyendo gracias a la formación continua.

En este caso, se pudo conocer, en sus estrategias metodológicas cuando se concretaban en las actividades que permitirían apropiarse del conocimiento, a través de procesos constructivos por parte de los alumnos. En ellas se integran tanto cuestiones disciplinares (aspectos teóricos y metodológicos) del saber a enseñar, como de aprendizaje (procesos reflexivos y análisis) donde los profesores por su trayecto los consideraron adecuados para ser incorporados en estas experiencias. 
Las estrategias metodológicas investigadas se posicionan dentro de un paradigma de cognición situada (Díaz Barriga, 2010), porque se destacó por la importancia de la actividad y el contexto para el aprendizaje. Porque se reconoce al aprendizaje como un proceso de enculturación en el cual los estudiantes se integran paulatinamente a un campo profesional, por ello, los alumnos aprenden en el contexto pertinente, en estos casos diseñados para ellos.

Desde esta visión situada, la enseñanza se centra en prácticas auténticas, las cuales son coherentes y significativas. Por la relevancia cultural de las actividades que participa el estudiante, porque los estudiantes aprenden implicándose con los desafíos que se les presentan en la práctica real en los diferentes campos del conocimiento.

Las estrategias metodológicas, posicionadas dentro de un paradigma de la cognición situada, propiciaron un sistema de evaluación procesual con el que los mismos docentes evaluaban el impacto que ocasionaban estas prácticas pedagógicas innovadoras. Desde luego que el impacto que ellos querían ocasionar se centraba en los aprendizajes de los estudiantes. Por ello se identificó que, para lograr una reflexión en estas prácticas pedagógicas innovadoras, se posicionaron en un tipo de evaluación procesual, que mediante el diálogo que se establecía entre profesores y alumnos, comprender los conocimientos aprendidos y así tomar decisiones en relación a una valoración. La evaluación fue una actividad compleja, que pretendió mejorar tanto la calidad de los aprendizajes de los alumnos como los métodos de enseñanza de los docentes. De más está decir que se necesita, desde ambos lados de esta relación, tener una apertura mental que posibilite la autocrítica y, por qué no, la autoevaluación (Tejedor \& Jornet, 2008).

Por medio de la evaluación, se identificó como sirvió al alumno para que éste corrobore sus aprendizajes y aprenda de sus errores. Fue imperiosa la devolución y la explicación de las equivocaciones. Esto permitió también detectar algún problema en la enseñanza. El autor anteriormente mencionado, nos ayudó a reconocer como la evaluación de desempeño que se realizó requirió de una tarea de observación de ciertas destrezas que el alumno fue construyendo a lo largo del curso. Lógicamente, este tipo de evaluación estuvo precedida por una metodología de enseñanza que hizo demostraciones didácticas de las destrezas requeridas, dentro de las competencias de acción que debe tener un futuro egresado.

El impacto que tuvieron estas prácticas equivale a la calidad de los aprendizajes, estos fueron satisfactorios y se construyeron aprendizaje gracias a un proceso de construcción personal de ellos, elaborando una representación personal del contenido de aprendizaje propuesto por el docente. Esto fue gracias a los conocimientos previos de los estudiantes que le sirvieron para conectar con lo nuevo brindado por el docente, esto fue resultado de un proceso activo de los estudiantes, donde el docente fue un guía.

Los estudiantes necesitaron realizaron un proceso de metacognición, para asegurar el control personal sobre sus conocimientos y los propios procesos durante el aprendizaje. El profesor y estas prácticas pedagógicas innovadoras se volvieron parte activa en el proceso de construcción de conocimiento que tiene como centro no a la materia, sino a los estudiantes que actuaron sobre el contenido. Así, esta concepción se caracteriza porque el alumnado es considerado constructor activo y no seres

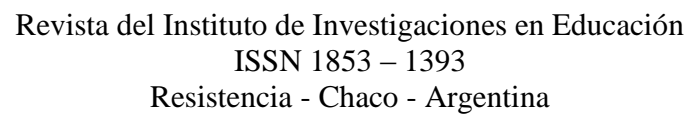


reactivos, y porque el profesorado se ocupa, realmente, de acompañarlos para la construcción de conocimientos (Mauri, 1999).

\section{A modo de cierre}

Entendemos que el impacto que tuvo la innovación fue directamente en los aprendizajes, no admitiendo al producto sin el proceso, es decir, el recorrido por el que el estudiante construye los conocimientos.

Al recorrer este proceso aprendían e iban cambiando no sólo la cantidad de información, sino la capacidad de comprender ese contenido. Desde esta perspectiva, resultó obvia la importancia de cómo se les enseñaba a los estudiantes a "aprender a aprender" y la de ayudarle a comprender que, cuando están aprendiendo, no se debe tener únicamente en cuenta aprender para pasar esa instancia y aprender ese contenido específico, sino también cómo organizarse para actuar de esa misma manera o mejor en situaciones futuras. En este sentido, queremos brindar dos consideraciones finales, para poder cerrar parcialmente esta presentación.

\section{- Cómo fue posible comprender estas experiencias...}

Comprender estos pasos fue complejo y enriquecedor para la tarea o el desafío que abordamos al momento de intentar sistematizar este tipo de prácticas valiosas para la docencia universitaria, ya que la indagación en esta dirección no sólo "observa" lo investigado, sino que modifica sustancialmente nuestra visión como investigadoras y docentes hacia la innovación, convencidas de que lo rutinario y monótono en la enseñanza debería ser modificado por nuevas formas de interacción entre el docente, alumno y el conocimiento.

Creemos en la importancia de haber conocido prácticas pedagógicas innovadoras por medio de la metodología de caso, con una actitud investigativa, que nos da la posibilidad de compartir con los pares para la transformación de las prácticas porque necesitamos darle sentido a la investigación cualitativa para impulsar las experiencias innovadoras en toda la Universidad, en todas las aulas.

\section{- Lo que nos Ilevamos...}

Una experiencia que nos ayudó a reconocer que la necesidad de innovar surge por un hecho de generosidad con el conocimiento, por desprenderse de la idea del rol protagónico de la clase por parte del docente. Por la intención de pensar que los estudiantes son el foco del aula, y que necesitan de los docentes para llegar a nuevos horizontes, la construcción de nuevos conocimientos. Innovar es ayudar, brindando herramientas para que los estudiantes controlen sus propios procesos durante el aprendizaje. Porque el profesor y estas prácticas pedagógicas innovadoras se vuelven parte activa en el proceso de construcción de conocimiento que tiene como centro no a la asignatura, sino a los estudiantes.

Nos llevamos la certeza que innovar en las prácticas pedagógicas universitarias, es habilitar a los estudiantes a intervenir sobre el contenido disciplinar. Innovar en las prácticas pedagógicas, es un gran desafío, no resulta sencillo, más cuando la cultura universitaria no está centrada en los estudiantes. Pero consideramos que es posible, porque estas prácticas son el motor para el desarrollo de las 
Universidades, un desarrollo que implica crecimiento económico y social. Para que esto se logre, necesitamos un equipo de docentes universitarios, que desplieguen creatividad para poder innovar en la Universidad, para poder fomentar en los estudiantes ganas de querer aprender más. Las experiencias que nos tocó profundizar, descubrimos que hay profesores universitarios, que lo están intentando; que comparten nuestro mismo compromiso con la práctica docente y la convicción sobre el rol docente innovador: ayudar a aprender.

\section{Bibliografía}

Alcalá, M. (2002). Concepciones epistemológicas de profesores de carrera de profesorados. Relaciones con la enseñanza en cátedras universitarias. Trabajo Correspondiente al período de investigación. Programa de Doctorado en Didáctica General y Didácticas Especiales. Universidad de León.

Altopiedi, M., \& Jiménez, L. (2010). Contextos difíciles e historias turbulentas como motores de la innovación. Profesorado. Revista de Currículum y Formación de Profesorado, 14 (1), 29-45.

Anijovich, R. \& Mora, S. (2009). Estrategias de enseñanza. Otra mirada al quehacer del aula. Buenos Aires: Aique.

Bergvall-Kåreborn, B., Eriksson, C., Ståhlbröst, A. \& Svensson, J. (2009). A milieu for innovation: defining living labs. In ISPIM Innovation Symposium. 06/12/0909/12/09 http://www.diva-portal.org/smash/record.jsf?pid=diva2\%3A1004774\&dswid=-218

Carbonell, J. (2002). El profesorado y la innovación educativa. En Cañal de León, P. (Coord.). La Innovación Educativa (pp. 11-26). Madrid: Akal.

Casas, M. \& Stojanovic, L. (2013). Innovación en la universidad iberoamericana. [artículo en línea]. Revista de Universidad y Sociedad del Conocimiento (RUSC), 10 (1), 61-74. http://www.redalyc.org/pdf/780/78025711005.pdf

Coller, X. (2005). Estudio de casos. Madrid: Centro de Investigaciones Sociológicas.

Gardner, H. (1992). Assessment in Context: The Alternative to Standardized Testing. In Gifford, B.R. and O'Connor, M. C. (Eds). Changing Assessments: Alternative Views of Aptitude, Achievement and Instruction (pp.77-121). Boston: Kluwer Academic Publishers.

Daniels, H. (2001). Vygotsky y la pedagogía. Buenos Aires: Paidós

Dabaieh, M., Lashin, M., \& Elbably, A. (2017). Going green in architectural education: An urban living lab experiment for a graduation green design studio in Saint Catherine, Egypt. Solar Energy, (144), 356-366.

Davini, M. (2008). Métodos activos de enseñanza y aprendizaje La integración del conocimiento y la práctica. Versión adaptada del Capítulo 5 (pp. 113-133).

Davini, M. (2009). Métodos de enseñanza. Didáctica general para maestros y profesores. Buenos Aires: Santillana. 
Demuth Mercado, P. (2011). El desarrollo del conocimiento didáctico en profesores universitarios principiantes: estudio de casos en el Departamento de Informática de la Universidad Nacional del Nordeste - Argentina. Trabajo de investigación inédito. DEA. Programa de Doctorado en Didáctica y Organización de Instituciones Educativas, Universidad de Sevilla, España.

Díaz Barriga Arceo, F. (2010). Los profesores ante las innovaciones curriculares. Revista Iberoamericana de Educación Superior, I (1), 37-57.

Engeström, Y. (1987). Learning by expanding. Cambridge: University Press.

Eggen, P., Kauchak, D. \& Libedinsky, M. (1999). Estrategias docentes: enseñanza de contenidos curriculares y desarrollo de habilidades de pensamiento. Fondo de cultura económica.

Fenstermacher, G. (1989). Tres aspectos de la filosofía de la investigación sobre la enseñanza. En Wittrock, M. (Comp.) La investigación de la enseñanza I. Enfoques, teorías y métodos (149-179). Barcelona: Paidós.

Gardner, H. (1992). Assessment in Context: The Alternative to Standardized Testing. In B. R. Gifford and M. C. O'Connor (Eds,) Changing Assessments: Alternative Views of Aptitude, Achievement and Instruction (pp. 77-121). Boston: Kluwer Academic Publishers.

Gewerc, A. \& Montero, M. (2000). ¿Profesor, Médico o científico? Un estudio de caso de catedráticos de la Universidad de Santiago de Compostela. Revista de Educación, (321), 371-398.

Jiménez, R. (2010). Formación por competencias en la enseñanza de la Arquitectura: ¿Un aporte? ¿Una necesidad? ¿O una moda? Revista Electrónica de Desarrollo de Competencias REDEC, 6 (2), 65-83.

Hernández Sampeiri. R., Fernández Collado, C., \& Baptista Lucio, P. (2003). Metodología de la investigación. México: Mc Graw-Hill.

Kunnari, I., \& Ilomäki, L. (2016). Reframing teachers' work for educational innovation. Innovations in Education and Teaching International, 53 (2), 167-178.

Libedinsky, M. (2001). La innovación en la enseñanza. Buenos Aires: Paidós.

Litwin, E. (2002). Prácticas con tecnologías. Cuadernos de Investigación Educativa, 1 (10), 43-51.

Marcelo, C. (2013). Las tecnologías para la innovación y la práctica docente. Revista Brasileira de Educação, 18, (52), 25-47.

Martín, E. (2009). Profesorado competente para formar alumnado competente: el reto del cambio docente. En Pozo, J. I. y Pérez, M. del P. (Coords.). Psicología del aprendizaje universitario: la formación en competencias. Madrid: Morata. 
Masseck, T. (2017). Living labs in architecture as innovation arenas within higher education institutions. Energy Procedia, (115), 383-389.

Michavila, F. (2009). La innovación educativa. Oportunidades y barreras. Arbor, 185 (Extra), 3-8.

Perkins, D. (1993). Person plus: "A distributed view of thinking and learning". En Salomon (Ed.) Distributed Cognitions. New York: Cambridge University Press.

Mauri, T. (1999). ¿Qué hace que el alumno y la alumna aprendan los contenidos escolares? En Coll, C. El constructivismo en el aula. (Cap. 4). Barcelona: Graó.

Meyer, C. (1992). What's the Difference between Authentic and Performance Assessment?. Educational Leadership, 49 (8), 39-40.

Porta, L., Yedaide, M., \& Álvarez, Z. (2016). Sentidos de la pasión. Revista Episteme, $6(1), 11-18$.

Porta, L., Sarasa, M., \& Alvarez, Z. (2011). Una experiencia en torno a la investigación sobre la enseñanza en el nivel superior. Revista de Educación, 2 (3), 181-210.

Pérez Ortega, I. (2016). La competencia mediática en el currículo escolar: ¿espacio para innovaciones educativas con tecnologías de la información y la comunicación? Innovación educativa, 16 (70), 61-83.

Pinos Medrano, H. (2015). Uso del método de aprendizaje basado en proyectos (ABP), para la carrera de Arquitectura. Revista Universidad y Sociedad [seriada en línea], 7 (3), 112-116.

Portero Ricol, A., \& Zurita, H. (2014). La experiencia del Taller en el Barrio El Astillero. Arquitectura y Urbanismo, 35 (3), 130-135.

Stake, R. (2007). Investigación con estudios de caso. Madrid: Morata.

Tardif, M. (2004). Los saberes del docente y su desarrollo profesional. Madrid: Narcea.

Tejedor, F. \& Jornet, J. (2008). La evaluación del profesorado universitario. Revista Electrónica de Investigación Educativa, Especial.

Yin, R. (1994). Case study research: Design and methods. California: Sage.

Viñao, A. (2002). Sistemas educativos, culturas escolares y reformas. Madrid: Morata.

Webster, F. A., \& Hopkins, D. W. (1996). Contributions from different microbial processes to $\mathrm{N} 2 \mathrm{O}$ emission from soil under different moisture regimes. Biology and Fertility of Soils, 22 (4), 331-335.

Zabalza, M. (2004). Innovación en la enseñanza universitaria. Contextos Educativos. Universidad de Santiago de Compostela. 